\title{
Dwell time-based stabilisation of switched linear delay systems using clock-dependent Lyapunov-Krasovskii functionals
}

\section{Ahmet Taha Koru, Akın Delibaşı \& Hitay Özbay}

To cite this article: Ahmet Taha Koru, Akın Delibașı \& Hitay Özbay (2020) Dwell time-based stabilisation of switched linear delay systems using clock-dependent Lyapunov-Krasovskii functionals, International Journal of Control, 93:5, 1172-1179, DOI: 10.1080/00207179.2018.1500036

To link to this article: https://doi.org/10.1080/00207179.2018.1500036

曲 Published online: 27 Jul 2018.

Submit your article to this journal

Џ Article views: 258

Q View related articles 두

View Crossmark data $\asymp$

Citing articles: 2 View citing articles 


\title{
Dwell time-based stabilisation of switched linear delay systems using clock-dependent Lyapunov-Krasovskii functionals
}

\author{
Ahmet Taha Koru (10 ${ }^{a}$, Akın Delibaşı ${ }^{b}$ and Hitay Özbay ${ }^{c}$ \\ ${ }^{a}$ Department of Mechatronics Engineering, Yıldız Technical University, Istanbul, Turkey; ${ }^{b}$ Department of Control and Automation Engineering, Yıldız \\ Technical University, Istanbul, Turkey; ${ }^{\mathrm{C}}$ Department of Electrical - Electronics Engineering, Bilkent University, Ankara, Turkey
}

\begin{abstract}
Dwell time stability conditions of the switched delay systems are derived using multiple clock-dependent Lyapunov-Krasovskii functionals. The corresponding conditions are approximated by both using piecewise linear functions and sum of squares polynomials. The upper bound of the dwell time is minimised using a combination of a bisection and a golden section search algorithm. Using the results obtained in the stability case, synthesis of dwell time minimiser controllers are presented. Some numerical examples are given to illustrate effectiveness of the proposed method, and its performance is compared with the existing approaches. The resulting values of the dwell time via the proposed technique show that the novel approach outperforms the previous ones.
\end{abstract}

\section{ARTICLE HISTORY}

Received 29 October 2017

Accepted 8 July 2018

\section{KEYWORDS}

Time delay; dwell time optimisation; clock-dependent Lyapunov; switched systems; SOS polynomials

\section{Introduction}

One of the main methods in the analysis of switched systems is restricting switching signals to a certain set. We can restrict the switching signals to those with the property that the time interval between any consecutive switching times is larger than a certain value, which is called the dwell time to guarantee the asymptotic stability of the switched systems. Readers are referred to Hespanha and Morse (1999), Yuan and Wu (2015), Mitra and Liberzon (2004), Lin and Antsaklis (2009), Goebel, Sanfelice, and Teel (2012) and references therein for further information on this topic.

There is a dwell time between switchings in many applications such as changing road conditions (dry, wet, dirt) of a car on the road, or two different dynamics of a teleoperation robotic system either contacting a tissue or not (Allerhand \& Shaked, 2011). Furthermore, fast switching can cause chattering problems in contrast to the control schemes where switching is restricted by a dwell time (Ishii \& Francis, 2001). As a result, stability analysis and stabilisation of switched systems with dwell time are becoming increasingly popular. Furthermore, dwell time-based stability and stabilisation methods are used in complex systems such as impulsive systems (Briat, 2017; Chen, Ruan, \& Zheng, 2017) and switching systems involving unstable subsystems (Zhao, Yin, Niu, \& Zheng, 2016).

Multiple Lyapunov functions method is a common technique in dealing with dwell time stability problems. In Geromel and Colaneri (2006), the dwell time is determined by constraints involving exponential terms, i.e. $\mathrm{e}^{A^{T} T_{D}} P_{i} \mathrm{e}^{A T_{D}}-P_{j} \succeq 0$. Another approach is employing piecewise linear Lyapunov functions which form convex sets in system matrices (Allerhand \& Shaked, 2011). In Xiang (2015), it is shown that stability criteria of Allerhand and Shaked (2011) and
Geromel and Colaneri (2006) are equivalent when a sufficiently large number of decision variables and linear matrix inequalities (LMIs) are included in the stability criterion of Allerhand and Shaked (2011). A nonconservative dwell time conditions using homogeneous polynomial Lyapunov functions and implementing them with sum of squares (SOS) polynomials are presented in Chesi, Colaneri, Geromel, Middleton and Shorten (2012). In Zhao, Shi, Yin, and Nguang (2017), tighter bounds on the dwell time are presented using multiple discontinuous Lyapunov functions for the switching systems involving stable and unstable subsystems. In Briat (2015b), the dwell time is represented with clock-dependent Lyapunov functions. The piecewise linear functions and approximations of SOS polynomials are compared. It is shown that the approximations of SOS polynomials decrease the computational complexity (Briat, 2016).

The literature is abounded with various approaches for the stability analysis of time-delay systems (see e.g. Briat, 2015a; Fridman, 2014; Gu, Kharitonov, \& Chen, 2003). Model transformation-based methods are common among the delaydependent stability methods. The method deals with the pointwise delay systems by transferring the system into a distributed delay system. Stability of the transformed system is a sufficient condition for the stability of the original system. As a result, model transformation-based methods are conservative since the analysis operates on the transformed system instead of the original system (Gu \& Niculescu, 2001). In more recent results, model transformations are not used, e.g. free-weighting matrices method (He, Wang, Xie, \& Lin, 2007; Wu, He, \& She, 2010). There are more recent results based on integral inequalities such as Jensen's inequality (Gouaisbaut \& Peaucelle, 2006), Wirtinger's integral inequality (Liu \& Fridman, 2012; Seuret \& 
Gouaisbaut, 2013; Seuret, Gouaisbaut, \& Fridman, 2013) and the Bessel-Legendre inequality (Liu, Seuret, \& Xia, 2017; Seuret \& Gouaisbaut, 2014). These new methods may further reduce the conservatism that is inherent in the stability analysis of time-delay systems.

A survey on the stability of switched delay systems is given in Mahmoud (2010). In Sun, Zhao, and Hill (2006) and Li, Gao, Agarwal, and Kaynak (2013), the average dwell time is represented with constraints involving exponential terms and bilinear matrix inequalities, which can be classified as a nondeterministic polynomial time decidable minimisation problem. In order to make the problem a tractable one, it is solved for a given average dwell time and tuned generalised eigenvalues rather than performing the dwell time minimisation. In the literature, there are limited studies that address the minimisation problem on dwell time (Çalışkan, Özbay, \& Niculescu, 2013; Koru, Delibaşı, \& Özbay, 2018; Yan \& Özbay, 2008; Yan, Özbay, \& Şansal, 2014). Model transformation methods are used in Çalışkan et al. (2013), Yan and Özbay (2008) and Yan et al. (2014), whereas a free-weighting matrices method, which does not include any model transformation, is used in Koru et al. (2018). In those works, none of the conditions contain a term that is formed as a product of transcendental function and its domain to avoid a non-convex representation of the problem. However, this leads to some conservatism: typically, the upper bound of the dwell time, leading to stability, is represented as

$$
T_{D}=h_{\max }+T^{*}
$$

where $h_{\max }$ is the maximum time delay among all of the subsystems and $T^{*}$ is the cost function. Hence, the minimum dwell time is at least $h_{\max }$ even for the systems sharing a common Lyapunov function which are known to be stable under arbitrary switching (Lin \& Antsaklis, 2009). This is a clear proof that the above-mentioned approaches cannot give the smallest possible dwell time in general.

In order to reduce the conservatism, the present paper derives stability conditions for switched linear delay systems using clock-dependent Lyapunov-Krasovskii functionals. As a result, the upper bound of the dwell time is represented without using the $h_{\max }$ term. The minimisation of the dwell time is formulated as a semidefinite programming problem by approximating the conditions in terms of piecewise linear functions and SOS polynomials. Upper bounds of the derivatives of the Lyapunov-Krasovskii functionals are found via Jensen's inequality and reciprocally convex functions (see Park, Ko, \& Jeong, 2011). Using the results of the stability case, the dwell time minimiser controller synthesis is presented.

\subsection{Notation}

The notation to be used in the paper is standard: $\mathbb{R}\left(\mathbb{R}^{+}, \mathbb{R}_{0}^{+}\right)$ stands for the set of real numbers (positive real numbers and non-negative real numbers), $\mathbb{S}^{n}$ denotes the set of symmetric matrices and $\mathbb{Z}^{+}$symbolises the set of positive integers. The identity matrices are denoted by $I$. We use $X \succ 0(\succcurlyeq, \prec, \preceq 0)$ to denote a positive definite (positive-semidefinite, negative definite and negative-semidefinite) matrix. The asterisk symbol $\left.{ }^{*}\right)$ denotes a complex conjugate transpose of a matrix and $x_{t}$ denotes the translation operator acting on the trajectory such as $x_{t}(\theta)=x(t+\theta)$ for some non-zero interval $\theta \in[-\tau, 0]$.

\section{Stability analysis}

In this section, we represent an upper bound of the dwell time for the switched delay systems in terms of clock-dependent Lyapunov-Krasovskii functionals. Afterwards, those conditions are approximated by both using SOS polynomials and piecewise linear functions.

Consider a class of switched delay systems given by

$$
\begin{array}{ll}
\dot{x}(t)=A_{\sigma(t)} x(t)+\bar{A}_{\sigma(t)} x(t-r(t)), & t \geq 0, \\
x(\theta)=\psi(\theta), & \forall \theta \in[-h, 0],
\end{array}
$$

where $x(t) \in \mathbb{R}^{n}$ is the state and $\sigma(t)$ is the piecewise constant switching signal such that $\sigma: \mathbb{R}_{0}^{+} \rightarrow \mathcal{I}$, the index set $\mathcal{I}:=\{1,2, \ldots, N\}$ represents subsystems, $N \in \mathbb{Z}^{+}$is the number of subsystems and initial condition $\psi(\cdot)$ belongs to the Banach space of continuous functions. Time delay, $r(t)$, is a time-varying differentiable function that satisfies

$$
0 \leq r(t) \leq h, \quad \dot{r}(t) \leq d \leq 1
$$

where $h$ and $d$ are positive constants. We introduce the notation

$$
\Sigma_{i}:=\left(A_{i}, \bar{A}_{i}\right) \in \mathbb{R}^{n \times n} \times \mathbb{R}^{n \times n}
$$

to denote the ith candidate subsystem of (1).

The following lemma will be used to bound the derivative of the Lyapunov-Krasovskii functional in the main theorem. This is based on Jensen's inequality and reciprocally convex combination of the resulting bound. The corresponding lemma is widely used in the time-delay system applications (Fridman, 2014; Park et al., 2011; Seuret et al., 2013).

Lemma 2.1: Let $R$ be a positive symmetric matrix-valued function satisfying

$$
\left[\begin{array}{cc}
R & S_{12} \\
* & R
\end{array}\right] \succeq 0 .
$$

Then, for a well-defined integration, the following inequality holds:

$$
\begin{aligned}
& -h \int_{t-h}^{t} \dot{x}^{\mathrm{T}}(s) R \dot{x}(s) \mathrm{d} s \\
& \leq \eta_{1}^{\mathrm{T}}\left[\begin{array}{ccc}
-R & S_{12} & R-S_{12} \\
* & -R & R-S_{12}^{\mathrm{T}} \\
* & * & -2 R+S_{12}+S_{12}^{\mathrm{T}}
\end{array}\right] \eta_{1},
\end{aligned}
$$

where

$$
\eta_{1}=\left[\begin{array}{lll}
x^{\mathrm{T}}(t) & x^{\mathrm{T}}(t-h) & x^{\mathrm{T}}(t-r(t))
\end{array}\right]^{\mathrm{T}} .
$$

Proof: Proof can be found in Yang, Zhang, Hui, and Wang (2012). 
Let us define the clock-dependent Lyapunov-Krasovskii functional for the subsystem $i$ as

$$
\begin{aligned}
V_{i}\left(t, x_{t}\right)= & x^{\mathrm{T}}(t) P_{i}(\tau) x(t)+\int_{t-r(t)}^{t} \mathrm{e}^{\gamma(s-t)} x^{\mathrm{T}}(s) Q_{i}(\tau) x(s) \mathrm{d} s \\
& +h \int_{-h}^{0} \int_{t+\theta}^{t} \mathrm{e}^{\gamma(s-t)} \dot{x}^{\mathrm{T}}(s) R_{i}(\tau) \dot{x}(s) \mathrm{d} s \mathrm{~d} \theta
\end{aligned}
$$

where $\tau=\min \left\{t-t_{k}, T_{D}\right\}, t_{k}$ is the last switching instant and $T_{D}$ is the dwell time. The Lyapunov-Krasovskii functional is clock-dependent in the time interval $t \in\left[t_{k}, t_{k}+T_{D}\right]$. After the dwell time, the parameters $P_{i}, Q_{i}, R_{i}$ are chosen to be constant such that $P_{i}(\tau)=P_{i}\left(T_{D}\right)$ for $t \geq t_{k}+T_{D}$. The Lyapunov-Krasovskii functional of system (1) is a multiple functional $\bar{V}\left(t, x_{t}, \sigma\right)=V_{\sigma}\left(t, x_{t}\right)$ and it jumps as the system switches. In the following theorem, we present the clockdependent dwell time conditions for which $V_{\sigma}\left(t, x_{t}\right)$ is decreasing for any active subsystem between switching instants and the jumps are non-increasing at the switching instants when they occur after the dwell time.

Theorem 2.2: For given $h \geq 0, d \in[0,1]$, tuning parameter $\gamma>0$ (see Remark 2.1) and the dwell time $T_{D}$, if there exist $n \times n$ symmetric matrix-valued functions $P_{i}(\tau) \succ 0, Q_{i}(\tau) \succ 0$, $R_{i}(\tau) \succ 0$, matrix-valued functions $S_{12 i}(\tau)$ such that the following inequalities are feasible:

$$
\begin{gathered}
\phi_{i}(\tau):=\left[\begin{array}{cccc}
\phi_{11 i}(\tau) & S_{12 i}(\tau) & \phi_{13 i}(\tau) & h A_{i}^{\mathrm{T}} R_{i}(\tau) \\
* & -\mathrm{e}^{-\gamma h} R_{i}(\tau) & \phi_{23 i}(\tau) & 0 \\
* & * & \phi_{33 i}(\tau) & h \bar{A}_{i}^{\mathrm{T}} R_{i}(\tau) \\
* & * & * & -R_{i}(\tau)
\end{array}\right] \prec 0, \\
\psi_{i}(\tau):=\left[\begin{array}{cc}
\mathrm{e}^{-\gamma h} R_{i}(\tau) & S_{12 i}(\tau) \\
* & \mathrm{e}^{-\gamma h} R_{i}(\tau)
\end{array}\right] \succeq 0, \\
\phi\left(T_{D}^{+}\right) \prec 0, \quad \psi\left(T_{D}\right) \succeq 0, \\
P_{i}\left(T_{D}\right)-P_{j}(0) \succeq 0, \\
Q_{i}\left(T_{D}\right)-Q_{j}(0) \succeq 0, \\
R_{i}\left(T_{D}\right)-R_{j}(0) \succeq 0, \\
\quad Q_{i}(\tau) \succeq 0, \quad \gamma R_{i}(\tau)-\dot{R}_{i}(\tau) \succeq 0
\end{gathered}
$$

for all $\tau \in\left[0, T_{D}\right]$, where

$$
\begin{aligned}
\phi_{11 i}(\tau)= & A_{i}^{\mathrm{T}} P_{i}(\tau)+P_{i}(\tau) A_{i}+\dot{P}_{i}(\tau)+Q_{i}(\tau)-\mathrm{e}^{-\gamma h} R_{i}(\tau), \\
\phi_{13 i}(\tau)= & P_{i}(\tau) \bar{A}_{i}+\mathrm{e}^{-\gamma h} R_{i}(\tau)-S_{12 i}(\tau), \\
\phi_{23 i}(\tau)= & \mathrm{e}^{-\gamma h} R_{i}(\tau)-S_{12 i}^{\mathrm{T}}(\tau), \\
\phi_{33 i}(\tau)= & -(1-d) \mathrm{e}^{-\gamma h} Q_{i}(\tau)-2 \mathrm{e}^{-\gamma h} R_{i}(\tau) \\
& +S_{12 i}(\tau)+S_{12 i}^{\mathrm{T}}(\tau),
\end{aligned}
$$

then system (1) is uniformly asymptotically stable for all switching signals with dwell time larger than or equal to $T_{D}$.
Proof: Derivative of $V$ given by (5) is

$$
\begin{aligned}
\dot{V}_{i}\left(t, x_{t}\right) \leq & 2 x^{\mathrm{T}}(t) P_{i}(\tau) \dot{x}(t)+x^{\mathrm{T}}(t) \dot{P}_{i}(\tau) x(t) \\
+ & x^{\mathrm{T}}(t) Q_{i}(\tau) x(t)-(1-d) \mathrm{e}^{-\gamma h} x^{\mathrm{T}}(t-r(t)) \\
& Q_{i}(\tau) x(t-r(t)) \\
+ & \int_{t-r(t)}^{t} \mathrm{e}^{\gamma(s-t)} x^{\mathrm{T}}(s)\left[\dot{Q}_{i}(\tau)-\gamma Q_{i}(\tau)\right](\tau) x(s) \mathrm{d} s \\
+ & h^{2} \dot{x}^{\mathrm{T}}(t) R_{i}(\tau) \dot{x}(t)-h \int_{t-h}^{t} \dot{x}^{\mathrm{T}}(s) \mathrm{e}^{-\gamma h} R_{i}(\tau) \dot{x}(s) \mathrm{d} s \\
+ & h \int_{-h}^{0} \int_{t+\theta}^{t} \mathrm{e}^{\gamma(s-t)} \dot{x}^{\mathrm{T}}(s)\left[\dot{R}_{i}(\tau)-\gamma R_{i}(\tau)\right]
\end{aligned}
$$$$
\dot{x}(s) \mathrm{d} s \mathrm{~d} \theta \text {. }
$$

The derivative of the Lyapunov-Krasovskii functional can be bounded as

$$
\begin{aligned}
\dot{V}_{i}\left(t, x_{t}\right) \leq & 2 x^{\mathrm{T}}(t) P_{i}(\tau) \dot{x}(t)+x^{\mathrm{T}}(t) \dot{P}_{i}(\tau) x(t) \\
& +\gamma_{i} x^{\mathrm{T}}(t) P(\tau) x(t)+x^{\mathrm{T}}(t) Q_{i}(\tau) x(t) \\
& -(1-d) \mathrm{e}^{-\gamma h} x^{\mathrm{T}}(t-r(t)) Q_{i}(\tau) x(t-r(t)) \\
& +h^{2} \dot{x}^{\mathrm{T}}(t) R_{i}(\tau) \dot{x}(t)-h \int_{t-h}^{t} \dot{x}^{\mathrm{T}}(s) \mathrm{e}^{-\gamma h} R_{i}(\tau) \dot{x}(s) \mathrm{d} s
\end{aligned}
$$

if (11) holds. After using Lemma 2.1 to bound the integral term, (12) can be represented in matrix form as

$$
\begin{gathered}
\dot{V}_{i}\left(t, x_{t}\right) \leq \eta_{1}^{\mathrm{T}}(t)\left[\begin{array}{ccc}
\phi_{11 i}(\tau) & S_{12 i}(\tau) & \phi_{13 i}(\tau) \\
* & -\mathrm{e}^{-\gamma h} R_{i}(\tau) & \phi_{23 i}(\tau) \\
* & * & \phi_{33 i}(\tau)
\end{array}\right] \\
\eta_{1}(t)+h^{2} \dot{x}^{T}(t) R_{i}(\tau) x(t),
\end{gathered}
$$

where $\eta_{1}$ is defined in (4). The Schur complement of the latter equation is (6). This condition guarantees the Lyapunov-Krasovskii functional in (5) is decreasing for any subsystem $i$ in time interval $t \in\left[t_{k}, t_{k}+T_{D}\right]$. Let $P_{i}(\tau)=P_{i}\left(T_{D}\right)$, $Q_{i}(\tau)=Q_{i}\left(T_{D}\right)$ and $R_{i}(\tau)=R_{i}\left(T_{D}\right)$ for all $t \geq t_{k}+T_{D}$ until the next switching instant $t_{j}$. As $\phi\left(T_{D}\right) \prec 0, \psi\left(T_{D}\right) \succeq 0$, the functional is decreasing for any non-switching subsystem $i$, $\forall t \geq t_{k}$.

Assume that the system is switched from the ith to the arbitrary jth subsystem at a switching instant $t_{j} \geq t_{k}+T_{D}$. For such an instant, the change in the Lyapunov-Krasovskii functional is

$$
\begin{aligned}
& V_{i}\left(t_{j}^{-}, x_{t}\right)-V_{j}\left(t_{j}^{+}, x_{t}\right) \\
& =x^{\mathrm{T}}(t)\left[P_{i}\left(T_{D}\right)-P_{j}(0)\right] x(t) \\
& \quad+\int_{t_{j}-r(t)}^{t_{j}} \mathrm{e}^{-\gamma\left(s-t_{j}\right)} x^{\mathrm{T}}(s)\left[Q_{i}\left(T_{D}\right)-Q_{j}(0)\right] x(s) \mathrm{d} s \\
& \quad+h \int_{-h}^{0} \int_{t_{j}+\theta}^{t_{j}} \mathrm{e}^{-\gamma\left(s-t_{j}\right)} \dot{x}^{\mathrm{T}}(s)\left[R_{i}\left(T_{D}\right)-R_{j}(0)\right] \dot{x}(s) \mathrm{d} s \mathrm{~d} \theta .
\end{aligned}
$$

The Lyapunov-Krasovskii functional cannot increase at switching instants as $V_{i}\left(t_{j}^{-}, x_{t}\right)-V_{j}\left(t_{j}^{+}, x_{t}\right) \geq 0$ if (8), (9) 
and (10) hold. Then, system (1) is uniformly asymptotically stable.

Remark 2.1: The parameter $\gamma$ does not represent the decay rate of the switched system. An initial $\gamma$ can be found or can be maximised via a bisection algorithm before calculating the dwell time using the conditions $\phi \prec 0$ and $\psi \succeq 0$ for constant parameters $P_{i}, Q_{i}, R_{i}$ and $S_{12 i}$. In the numerical examples, we found the upper and lower bounds of the $\gamma$ for a large dwell time. The minimum dwell time is searched via a golden section algorithm over $\gamma$, and a bisection algorithm over $T_{D}$ for each given $\gamma$.

\subsection{Euler-based discretisation}

The conditions in Theorem 2.2 can be approximated by the $K$ many piecewise linear functions. For given scalars $K$ and $T_{D}$, time intervals can be chosen equidistant as

$$
\delta=\frac{T_{D}}{K} .
$$

Let us define $P_{i, k}, Q_{i, k}, R_{i, k}, S_{12 i, k}, k=0, \ldots, K$ and

$$
P_{i}(\tau)=\left\{\begin{array}{l}
\left(1-\frac{t-k \delta}{\delta}\right) P_{i, k}+\left(\frac{t-k \delta}{\delta}\right) P_{i, k+1}, \\
\tau \in[k \delta,(k+1) \delta], P_{i, K}, \tau \geq T_{D},
\end{array}\right.
$$

and the derivative is

$$
\dot{P}_{i}(\tau) \begin{cases}\frac{1}{\delta}\left(P_{i, k+1}-P_{i, k}\right), & \tau \in[k \delta,(k+1) \delta), \\ 0, & \tau \geq T_{D} .\end{cases}
$$

Let $Q_{i}(\tau), R_{i}(\tau)$ and $S_{12 i}(\tau)$ be defined similar to $P_{i}(\tau)$.

Note that, there are discontinuities in the derivatives at all $k \delta$ instants,

$$
\begin{aligned}
& \dot{P}_{i}\left(k \delta^{-}\right)=\left(P_{i, k}-P_{i, k-1}\right) / \delta, \\
& \dot{P}_{i}\left(k \delta^{+}\right)=\left(P_{i, k+1}-P_{i, k}\right) / \delta .
\end{aligned}
$$

With those definitions, all of the conditions in Theorem 2.2 are LMIs given as

$$
\begin{array}{ll}
P_{i, k} \succ 0, \quad Q_{i, k} \succ 0, \quad R_{i, k} \succ 0, & \forall k=0, \ldots, K, \\
\phi_{i}\left(k \delta^{+}\right) \prec 0, \quad \psi_{i}(k \delta) \succeq 0, & \forall k=0, \ldots, K, \\
\phi_{i}\left(k \delta^{-}\right) \prec 0, & \forall k=1, \ldots, K, \\
P_{i, K}-P_{j, 0} \succeq 0, & j \neq i, \\
Q_{i, K}-Q_{j, 0} \succeq 0, & j \neq i, \\
R_{i, K}-R_{j, 0} \succeq 0, & j \neq i, \\
\gamma_{i} Q_{i, k}-1 / \delta\left(Q_{i, k+1}-Q_{i, k}\right), & \forall k=0, \ldots, K-1, \\
\gamma_{i} Q_{i, k+1}-1 / \delta\left(Q_{i, k+1}-Q_{i, k}\right), & \forall k=0, \ldots, K-1, \\
\gamma_{i} R_{i, k}-1 / \delta\left(R_{i, k+1}-R_{i, k}\right), & \forall k=0, \ldots, K-1, \\
\gamma_{i} R_{i, k+1}-1 / \delta\left(R_{i, k+1}-R_{i, k}\right), & \forall k=0, \ldots, K-1 .
\end{array}
$$

By virtue of the proposed representation for the stability condition, one can derive the dwell time by using a bisection algorithm. In the numerical examples, we solved the problems by using SeDuMi (Sturm, 1999).
A discussion on the effect of the selection of $K$ for the nondelayed case can be found in Xiang (2015). As $K$ increases, the results are less conservative. On the other hand, the increment in $K$ leads to high computational cost.

\subsection{SOS programming}

Clock-dependent conditions can be approximated well by using SOS polynomials with a lower computational cost when it is compared to Euler-based discretisation. For more details and dwell time calculation for the non-delayed switching systems, see Briat (2015b). In this section, we only present the SOS program with similar methods to those of the corresponding paper.

The following theorem is used to relax the 'whenever' conditions such as (6), (7) and (11) in SOS programs, similar to the $\mathrm{S}$-procedure in LMI techniques.

Theorem 2.3 (Theorem 4 of Briat (2015b)): Let the semialgebraic set be $\mathcal{G}:=\left\{\tau \in \mathbb{R} \mid g(\tau):=-\tau\left(T_{D}-\tau\right) \leq 0\right\}$. A symmetric matrix-valued function $Q(\tau) \succeq 0$ over $\mathcal{G}$ if and only if there exists an SOS matrix $S(\tau) \in \mathbb{S}^{n}$ such that the matrix $Q(\tau)+S(\tau) g(\tau)$ is SOS.

The following SOS program is associated with the conditions of Theorem 2.2:

Find polynomials $P_{i}, Q_{i}, R_{i}:\left[0, T_{D}\right] \rightarrow \mathbb{S}^{n}$,

$$
\begin{aligned}
& S_{12 i}:\left[0, T_{D}\right] \rightarrow \mathbb{R}^{n \times n}, \\
& \Gamma_{i}^{\dot{Q}}, \Gamma_{i}^{\dot{R}}:\left[0, T_{D}\right] \rightarrow \mathbb{S}^{n}, \\
& \Gamma_{i}^{\phi}:\left[0, T_{D}\right] \rightarrow \mathbb{S}^{4 n}, \\
& \Gamma_{i}^{\psi}:\left[0, T_{D}\right] \rightarrow \mathbb{S}^{2 n},
\end{aligned}
$$

such that $P_{i}, Q_{i}, R_{i}, \Gamma_{i}^{\dot{Q}}, \Gamma_{i}^{\dot{R}}, \Gamma_{i}^{\phi}, \Gamma_{i}^{\psi}$ are SOS

$$
\begin{aligned}
& \gamma_{i} Q_{i}(\tau)-\dot{Q}_{i}(\tau)-\Gamma_{i}^{\dot{Q}}(\tau) \tau\left(T_{D}-\tau\right) \text { is SOS } \\
& \gamma_{i} R_{i}(\tau)-\dot{R}_{i}(\tau)-\Gamma_{i}^{\dot{R}}(\tau) \tau\left(T_{D}-\tau\right) \text { is SOS } \\
& -\phi_{i}(\tau)-\Gamma_{i}^{\phi}(\tau) \tau\left(T_{D}-\tau\right)-\epsilon I \text { is SOS } \\
& \psi_{i}(\tau)-\Gamma_{i}^{\psi}(\tau) \tau\left(T_{D}-\tau\right) \text { is SOS } \\
& -\phi_{i}\left(T_{D}\right)-\epsilon I \succ 0, \quad \psi_{i}\left(T_{D}\right) \succeq 0 . \\
& P_{i}\left(T_{D}\right)-P_{j}(0) \succeq 0, \\
& Q_{i}\left(T_{D}\right)-Q_{j}(0) \succeq 0, \\
& R_{i}\left(T_{D}\right)-R_{j}(0) \succeq 0 .
\end{aligned}
$$

Combination of SOSTools (Prajna, Papachristodoulou, \& Parrilo, 2002) and SeDuMi can be used to solve such SOS programs in Matlab environment.

\subsection{Numerical examples for the stability analysis}

In this section, the examples are taken from published papers for comparison purposes. Examples 2.1, 2.2 and 2.3 can be found in Çalışkan et al. (2013), Chen and Zheng (2010) and Koru et al. (2018), respectively. 
$T_{d}$ vs $h$ for $d=0$

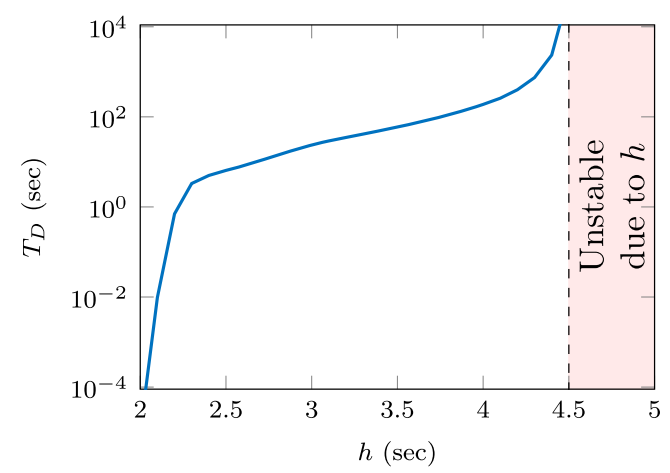

$T_{d}$ vs $d$ for $h=2.2$

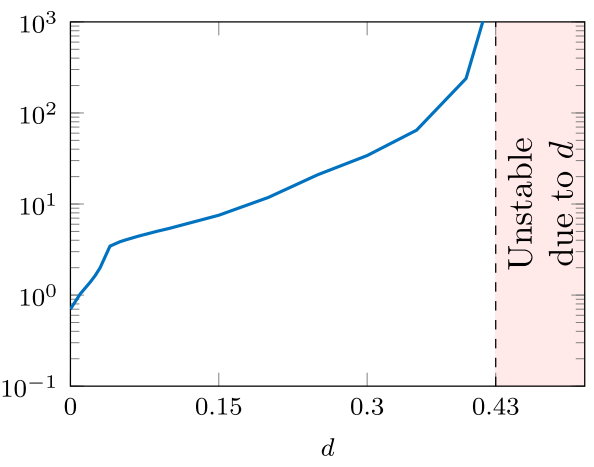

Figure 1. Dwell time results for various time delay and delay derivative upper bounds of Example 2.1. When $d=0$, the system admits common Lyapunov functionals for $h<2.13 \mathrm{~s}$.

Example 2.1: Let $\Sigma_{1}$ and $\Sigma_{2}$ be

$$
\begin{array}{ll}
A_{1}=\left[\begin{array}{cc}
-2 & 0 \\
0 & -0.9
\end{array}\right], \quad \bar{A}_{1}=\left[\begin{array}{cc}
-1 & 0 \\
-0.5 & -1
\end{array}\right], \\
A_{2}=\left[\begin{array}{cc}
-1 & 0.5 \\
0 & -1
\end{array}\right], \quad \bar{A}_{2}=\left[\begin{array}{cc}
-1 & 0 \\
0.1 & -1
\end{array}\right] .
\end{array}
$$

Upper bounds for the time delay are $h=0.6$ and $d=0$. The resulting dwell time is $T_{D}=7.6 \times 10^{-5} \mathrm{~s}$ for $\gamma=1.757$. For those parameters, it is shown by LMI feasibility tests that the switched delay system admits common Lyapunov functionals in the form

$$
\begin{aligned}
V(x, t)= & x^{\mathrm{T}}(t) P x(t)+\int_{t-r(t)}^{t} x^{\mathrm{T}}(s) Q x(s) \mathrm{d} s \\
& +\int_{-h}^{0} \int_{t+\theta}^{t} \dot{x}(s) R \dot{x}(s) \mathrm{d} s \mathrm{~d} \theta
\end{aligned}
$$

up to $h<2.13 \mathrm{~s}$.

Dwell time values for different $h$ and $d$ values are illustrated in Figure 1 with the proposed algorithm. As seen in the figure, the required dwell time is increasing as the time delay increases. Since subsystems are not stable independent of delay, it is not possible to stabilise systems with slow switching strategies if time delay is larger than a certain value, which is approximately $4.5 \mathrm{~s}$.

Example 2.2: This example is Case 2 of the first example of the paper Chen and Zheng (2010). Let $\Sigma_{1}$ be

$$
A_{1}=\left[\begin{array}{cc}
0 & 1 \\
-10 & -1
\end{array}\right], \quad \bar{A}_{1}=\left[\begin{array}{cc}
0.1 & 0 \\
-0.01 & 0.05
\end{array}\right],
$$

and $\Sigma_{2}$ be:

$$
A_{2}=\left[\begin{array}{cc}
0 & 1 \\
-0.1 & -0.5
\end{array}\right], \quad \bar{A}_{2}=\left[\begin{array}{cc}
0.02 & 0 \\
-0.01 & 0.02
\end{array}\right],
$$

$h=20.25, d=0$. The resulting dwell time is $6.671 \mathrm{~s}$ for $\gamma=$ 0.0133;

Example 2.3: This example is a slightly modified version of Example 1.1 of Sun and Ge (2011). In the example, a system is not guaranteed to be stable under arbitrary switching.
Let $\Sigma_{1}$ be

$$
A_{1}=\left[\begin{array}{cc}
-0.05 & 1.1 \\
-0.9 & -1
\end{array}\right], \quad \bar{A}_{1}=\left[\begin{array}{cc}
0.05 & -0.1 \\
-0.1 & 0
\end{array}\right],
$$

and $\Sigma_{2}$ be

$$
A_{2}=\left[\begin{array}{cc}
-0.05 & 1 \\
-150 & -50
\end{array}\right], \quad \bar{A}_{2}=\left[\begin{array}{cc}
0.05 & 0 \\
-1 & -1
\end{array}\right] .
$$

For $h=0.05$ and $d=0.2$, the resulting dwell time is $T_{D}=0.198 \mathrm{~s}$ for $\gamma=21.58$. Simulation results for two different switching scenarios can be seen in Figure 2. In the figure, it can be seen that for the switching signal with a dwell time $0.06 \mathrm{~s}$ (on the left bottom of the figure), system behaviour is unstable. On the other hand, the dwell time is $0.198 \mathrm{~s}$ for the switching signal on the right bottom and the resulting behaviour is stable.

Comparison of the present paper with previous works for all examples can be seen in Table 1. For Example 2.1, the system admits a common Lyapunov functions and the presented algorithm calculates a minimum dwell time less than $1 \times 10^{-4}$. For Examples 2.2 and 2.3, there is a significant decrease in the calculated upper bound for the dwell time. It can be seen from the table that the presented algorithm outperforms the other works.

\section{Controller design}

Consider a class of switched delay systems given by

$$
\tilde{\Sigma}_{\sigma(t)}: \begin{cases}\dot{x}(t)=A_{\sigma(t)} x(t) & \\ +\bar{A}_{\sigma(t)} x\left(t-r_{\sigma(t)}(t)\right)+B_{\sigma(t)} u(t), & t \geq 0, \\ x(\theta)=\varphi(\theta), & \forall \theta \in[-h, 0] .\end{cases}
$$

We introduce the trio

$$
\tilde{\Sigma}_{i}:=\left(A_{i}, \bar{A}_{i}, B_{i}\right) \in \mathbb{R}^{n \times n} \times \mathbb{R}^{n \times n} \times \mathbb{R}^{n \times m}
$$

to describe the $i$ th candidate subsystem.

Theorem 3.1: For given $h \geq 0, d \in[0,1]$, tuning parameters $\epsilon_{Q}, \epsilon_{R}$ and the dwell time $T_{D}$, if there exist $n \times n$ symmetric matrix-valued functions $\bar{P}_{i}(\tau) \succeq 0$, matrix-valued functions 

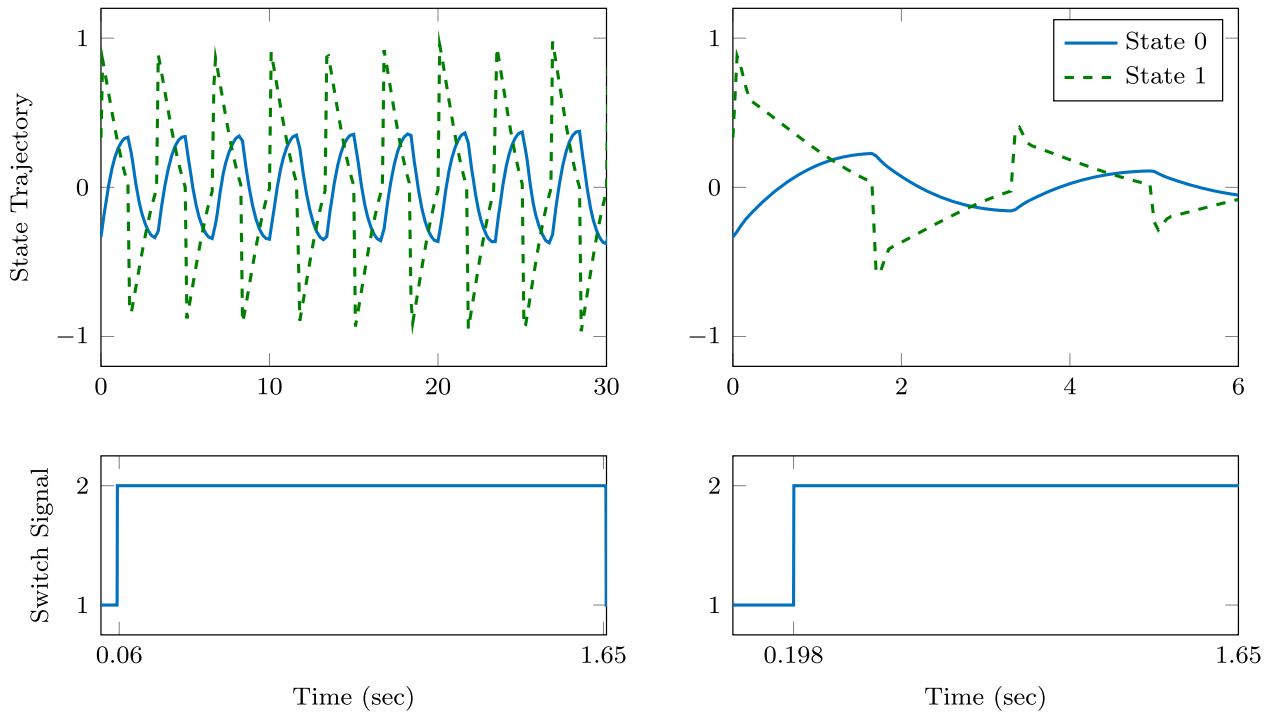

Figure 2. Simulation results of Example 2.3 for two different switching scenarios.

Table 1. Comparative dwell time results of Examples 2.1, 2.2 and 2.3.

\begin{tabular}{lccc}
\hline & Example 2.1 & Example 2.2 & Example 2.3 \\
\hline Chen and Zheng (2010) & - & $14.79 \mathrm{~s}$ & - \\
Yan and Özbay (2008) & $6.51 \mathrm{~s}$ & - & - \\
Çalışkan et al. (2013) & $3.4 \mathrm{~s}$ & - & - \\
Koru et al. (2018) & $1.11 \mathrm{~s}$ & $16.60 \mathrm{~s}$ & $1.68 \mathrm{~s}$ \\
Present paper & $7.6 \times 10^{-5} \mathrm{~s}$ & $6.671 \mathrm{~s}$ & $0.198 \mathrm{~s}$ \\
\hline
\end{tabular}

$L_{i}(\tau), \bar{S}_{12}(\tau)$ with appropriate dimensions such that following linear matrix-valued function inequalities are feasible:

$$
\begin{gathered}
\bar{\phi}_{i}(\tau):=\left[\begin{array}{ccc}
\bar{\phi}_{11 i}(\tau) & \bar{S}_{12 i}(\tau) & \bar{\phi}_{13 i}(\tau) \\
* & -\epsilon_{R} \mathrm{e}^{-\gamma h} \bar{P}_{i}(\tau) & \bar{\phi}_{23 i}(\tau) \\
* & * & \bar{\phi}_{33 i}(\tau) \\
* & * & * \\
h \epsilon_{R}\left(\bar{P}_{i}(\tau) A_{i}^{\mathrm{T}}+L_{i}^{\mathrm{T}}(\tau) B_{i}^{\mathrm{T}}\right) \\
0 \\
h \epsilon_{R} \bar{P}_{i}(\tau) \bar{A}_{i}^{\mathrm{T}} \\
-\epsilon_{R} \bar{P}_{i}(\tau)
\end{array}\right] \prec 0, \\
\bar{\psi}_{i}(\tau):=\left[\begin{array}{c}
\epsilon_{R} \mathrm{e}^{-\gamma h} \bar{P}_{i}(\tau) \\
* \\
\bar{S}_{12 i}(\tau) \\
\epsilon_{R} \mathrm{e}^{-\gamma h} \bar{P}_{i}(\tau)
\end{array}\right] \succeq 0, \\
\phi\left(T_{D}^{+}\right) \prec 0, \quad \psi\left(T_{D}\right) \succeq 0, \\
-\bar{P}_{i}\left(T_{D}\right)+\bar{P}_{j}(0) \succeq 0, \\
\gamma \bar{P}_{i}(\tau)+\dot{\bar{P}}_{i}(\tau) \succeq 0
\end{gathered}
$$

for all $\tau \in\left[0, T_{D}\right]$, where

$$
\begin{aligned}
\bar{\phi}_{11 i}(\tau)= & \bar{P}_{i}(\tau) A_{i}^{\mathrm{T}}+A_{i} \bar{P}_{i}(\tau)+B_{i} L_{i}(\tau)+L_{i}^{\mathrm{T}}(\tau) B_{i}^{\mathrm{T}}-\dot{\bar{P}}_{i}(\tau) \\
& +\bar{Q}_{i}(\tau)-\epsilon_{R} \mathrm{e}^{-\gamma h} \bar{P}_{i}(\tau), \\
\bar{\phi}_{13 i}(\tau)= & \bar{A}_{i} \bar{P}_{i}(\tau)+\epsilon_{R} \mathrm{e}^{-\gamma h} \bar{P}_{i}(\tau)-\bar{S}_{12 i}(\tau),
\end{aligned}
$$

$$
\begin{aligned}
\bar{\phi}_{23 i}(\tau)= & \epsilon_{R} \mathrm{e}^{-\gamma h} \bar{P}_{i}(\tau)-\bar{S}_{12 i}^{\mathrm{T}}(\tau), \\
\bar{\phi}_{33 i}(\tau)= & -(1-d) \mathrm{e}^{-\gamma h} \bar{Q}_{i}(\tau)-2 \epsilon_{R} \mathrm{e}^{-\gamma h} \bar{P}_{i}(\tau) \\
& +\bar{S}_{12 i}(\tau)+\bar{S}_{12 i}^{\mathrm{T}}(\tau),
\end{aligned}
$$

then system (13) can be stabilised by the control law $u_{i}(t)=$ $G_{i}(\tau) x(t)$ with the controller gains $G_{i}(\tau)=L_{i}(\tau) \bar{P}_{i}^{-1}(\tau)$ for all switching signals with dwell time larger than $T_{D}$.

Proof: For the memoryless feedback $G_{i}(\tau) x(t)$, we replace $A_{i}$ with $A_{i}+B_{i} G_{i}(\tau)$. If we pre- and post-multiply (6) by $\operatorname{diag}\left[P_{i}^{-1}(\tau), P_{i}^{-1}(\tau), P_{i}^{-1}(\tau), R_{i}^{-1}(\tau)\right]$ and $(7)$ by $\operatorname{diag}\left[P_{i}^{-1}(\tau)\right.$, $\left.P_{i}^{-1}(\tau)\right]$, make the change of variables $\bar{P}_{i}(\tau)=P_{i}^{-1}(\tau), \bar{S}_{12 i}(\tau)=$ $P_{i}^{-1}(\tau), S_{12 i}(\tau) P_{i}^{-1}(\tau)$ and choose $Q_{i}(\tau)=\epsilon_{Q} P_{i}(\tau), R_{i}(\tau)=$ $\epsilon_{R} P_{i}(\tau)$, then we derive the conditions.

Similar to the stability case, the conditions of Theorem 3.1 can be approximated by piecewise linear functions and SOS polynomials.

\subsection{Numerical examples for the stabilisation}

Example 3.1: This example is from Yan et al. (2014). In the corresponding paper, stabilisation of a linear time-varying (LTV) system is guaranteed with a switching controller. In order to

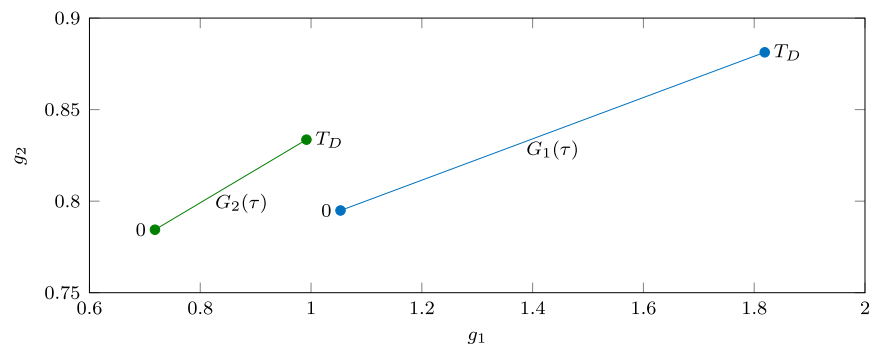

Figure 3. Time-varying controllers for Example 3.1. Controller starts at switching instant $t_{j}$ and reaches to value at $t_{j}+T_{D}$ and stays there until the next switching instant. 

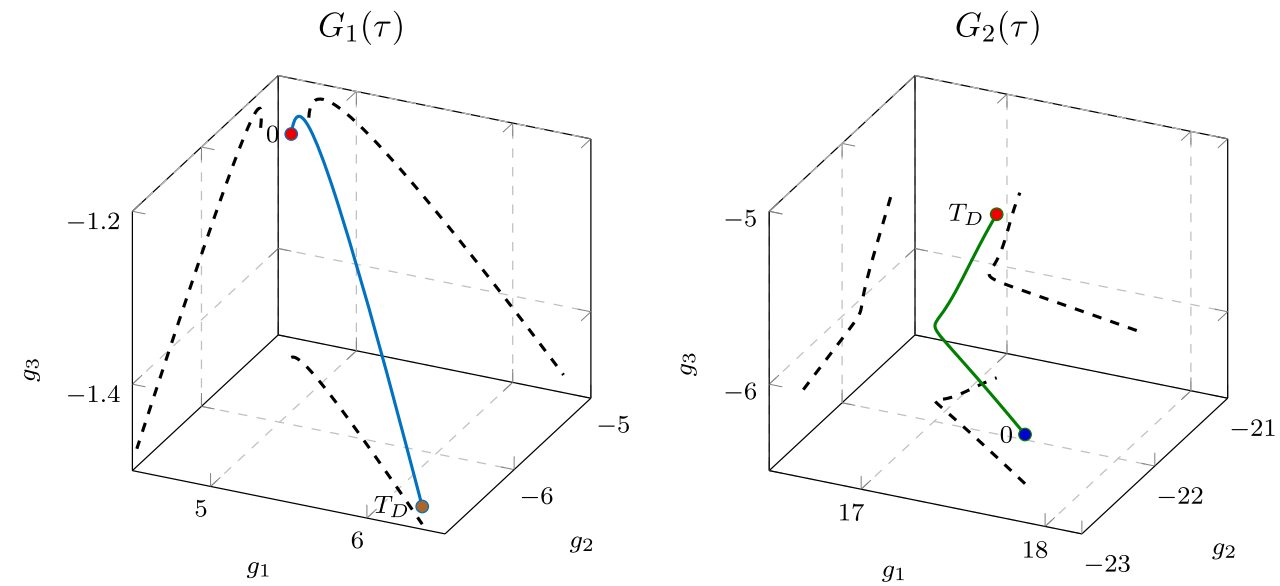

Figure 4. Time-varying controllers for Example 3.2, $g_{i}$ is the ith element of the controller gains. Dashed black lines are the 2D projections of the controller gains on to planes.

achieve that, the LTV system is represented as a switching delay system with two nominal subsystems and uncertainty bounds are determined. Then, controllers are designed with robust stability conditions. Synthesised controllers are

$$
G_{1}=[0.9681,0.0465], \quad G_{2}=[-0.2708,0.3715]
$$

and the resulting dwell time is found to be $0.92 \mathrm{~s}$. In this paper, we only considered the nominal subsystems of the switching delay system representation. The two nominal subsystems are defined as

$$
\begin{aligned}
& A_{1}=\left[\begin{array}{cc}
-3 & -1 \\
-1 & -1.9
\end{array}\right], \quad \bar{A}_{1}=\left[\begin{array}{cc}
-1 & 0 \\
-0.45 & -1
\end{array}\right], \\
& B_{1}=\left[\begin{array}{l}
1 \\
1
\end{array}\right] \text {, } \\
& A_{2}=\left[\begin{array}{cc}
-2 & -0.5 \\
-1 & -2
\end{array}\right], \quad \bar{A}_{2}=\left[\begin{array}{cc}
-1 & 0 \\
0.05 & -1
\end{array}\right] \text {, } \\
& B_{2}=\left[\begin{array}{l}
1 \\
1
\end{array}\right] \text {, }
\end{aligned}
$$

and $h=0.2 \mathrm{~s}$. Resulting controllers can be seen in Figure 3, where $g_{1}$ and $g_{2}$ are the first and second entries of the controller gain $G$. The resulting dwell time is $T_{d}=7.58 \times 10^{-9} \mathrm{~s}$. In this example, the pre-chosen parameter $K=2$ and the algorithm finds common Lyapunov functionals as

$$
\bar{P}_{i, k}=\left[\begin{array}{cc}
1.02 & -0.02 \\
-0.02 & 1.01
\end{array}\right]:=\bar{P}, \quad \forall i \in\{1,2\}, \quad \forall k=\{1,2\} .
$$

Since $G_{i}(\tau)=L_{i}(\tau) \bar{P}^{-1}$, the controllers are affine functions. Hence, the switched delay system is stable under arbitrary switching even when controlled with a static feedback controller $G_{i}=L_{i}(\tau) \bar{P}^{-1}$ for any $\tau \in\left[0, T_{D}\right]$.

Example 3.2: This example is a slightly modified version of the example of Yuan and $\mathrm{Wu}$ (2015), where the switched system in question is a non-delayed system which does not admit a common Lyapunov function. In the corresponding example,
Table 2. Comparative dwell time results of Examples 3.1 and 3.2

\begin{tabular}{lcc}
\hline & Example 3.1 & Example 3.2 \\
\hline Yan et al. (2014) & $0.92 \mathrm{~s}$ & \\
Koru et al. (2018) & $0.49 \mathrm{~s}$ & $0.58 \mathrm{~s}$ \\
Present Paper & $7.58 \times 10^{-9} \mathrm{~s}$ & $0.33 \mathrm{~s}$ \\
\hline
\end{tabular}

the switched linear plant is in the form:

$$
\dot{x}=A_{0, \sigma(t)} x+B_{0, \sigma(t)} w+B_{1, \sigma(t)} \mathcal{u},
$$

where $w$ is the disturbance,

$$
\begin{array}{lll}
A_{0,1}=\left[\begin{array}{ccc}
0.5108 & -0.9147 & -0.2 \\
-0.6563 & 0.1798 & 0.113 \\
0.881 & -0.7841 & 0.1
\end{array}\right], & B_{1,1}=\left[\begin{array}{c}
0.3257 \\
1.2963 \\
2.43
\end{array}\right], \\
A_{0,2}=\left[\begin{array}{ccc}
-0.125 & -0.9833 & -0.34 \\
-0.5305 & 0.3848 & 0.58 \\
1.0306 & 0.6521 & 0.1
\end{array}\right], & B_{1,2}=\left[\begin{array}{c}
1.0992 \\
0.6532 \\
3.5
\end{array}\right] .
\end{array}
$$

By using $A_{0, i}$ and $B_{1, i}$, we generated our example. Let $\Sigma_{1}$ and $\Sigma_{2}$ be

$$
\begin{array}{lll}
A_{1}=(1-\lambda) \cdot A_{0,1}, & \bar{A}_{1}=\lambda \cdot A_{0,1}, & B_{1}=B_{1,1}, \\
A_{2}=(1-\lambda) \cdot A_{0,2}, & \bar{A}_{2}=\lambda \cdot A_{0,2}, & B_{2}=B_{1,2} .
\end{array}
$$

For $\lambda=0.2$ and $h=0.45 \mathrm{~s}$, we choose $\epsilon_{Q}=0.1, \epsilon_{R}=0.6$, $\gamma=1$; the resulting dwell time is $0.33 \mathrm{~s}$ for degree $6 \mathrm{SOS}$ polynomials. The synthesised controllers can be seen in Figure 4 .

The comparison of the presented method with previous works can be seen in Table 2. There is a significant decrease in the dwell time results.

\section{Conclusions}

In this paper, clock-dependent Lyapunov-Krasovskii functionals are used to derive the dwell time stability conditions of switched delay systems. Feasibility of the represented dwell time is solved using SDP techniques and minimised using a combination of a bisection and a golden section search algorithm.

Then, the conditions to synthesise the dwell time minimiser controllers are derived. Numerical examples show that the presented algorithms outperform previous works for both the stability and the stabilisation case. 


\section{Acknowledgments}

The authors would like to thank anonymous reviewers for their suggestions that led to improvements in the paper. They would also like to thank $\mathrm{Dr}$ Corentin Briat for sharing his Matlab codes.

\section{Disclosure statement}

No potential conflict of interest was reported by the authors.

\section{ORCID}

Ahmet Taha Koru (D) http://orcid.org/0000-0001-8191-2324

\section{References}

Allerhand, L., \& Shaked, U. (2011). Robust stability and stabilization of linear switched systems with dwell time. IEEE Transactions on Automatic Control, 56(2), 381-386.

Briat, C. (2015a). Linear parameter-varying and time-delay systems: Analysis, observation, filtering \& control. Heidelberg: Springer.

Briat, C. (2015b). Convex conditions for robust stabilization of uncertain switched systems with guaranteed minimum and mode-dependent dwell-time. Systems \& Control Letters, 78, 63-72.

Briat, C. (2016). Theoretical and numerical comparisons of looped functionals and clock-dependent Lyapunov functions - the case of periodic and pseudo-periodic systems with impulses. International Journal of Robust and Nonlinear Control, 26(10), 2232-2255.

Briat, C. (2017). Dwell-time stability and stabilization conditions for linear positive impulsive and switched systems. Nonlinear Analysis: Hybrid Systems, 24, 198-226.

Çalışkan, S. Y., Özbay, H., \& Niculescu, S.-I. (2013). Dwell-time computation for stability of switched systems with time delays. IET Control Theory \& Applications, 7(10), 1422-1428.

Chen, W.-H., Ruan, Z., \& Zheng, W. X. (2017). Stability and $\mathcal{L}_{2}$-gain analysis for impulsive delay systems: An impulse-time-dependent discretized Lyapunov functional method. Automatica, 86, 129-137.

Chen, W.-H., \& Zheng, W. X. (2010). Delay-independent minimum dwell time for exponential stability of uncertain switched delay systems. IEEE Transactions on Automatic Control, 55(10), 2406-2413.

Chesi, G., Colaneri, P., Geromel, J. C., Middleton, R., \& Shorten, R. (2012). A nonconservative LMI condition for stability of switched systems with guaranteed dwell time. IEEE Transactions on Automatic Control, 57(5), $1297-1302$.

Fridman, E. (2014). Tutorial on Lyapunov-based methods for time-delay systems. European Journal of Control, 20(6), 271-283.

Geromel, J. C., \& Colaneri, P. (2006). Stability and stabilization of continuous time switched linear systems. SIAM Journal on Control and Optimization, 45(5), 1915-1930.

Goebel, R., Sanfelice, R. G., \& Teel, A. R. (2012). Hybrid dynamical systems: Modeling, stability, and robustness. Princeton, NJ, USA: Princeton University Press.

Gouaisbaut, F., \& Peaucelle, D. (2006). Delay-dependent robust stability of time delay systems. In The 5th IFAC Symposium on Robust Control Design, Toulouse, France.

Gu, K., Kharitonov, V., \& Chen, J. (2003). Stability of time-delay systems. Boston: Birkhauser.

Gu, K., \& Niculescu, S.-I. (2001). Further remarks on additional dynamics in various model transformations of linear delay systems. IEEE Transactions on Automatic Control, 46(3), 497-500.

He, Y., Wang, Q.-G., Xie, L., \& Lin, C. (Feb 2007). Further improvement of free-weighting matrices technique for systems with time-varying delay. IEEE Transactions on Automatic Control, 52(2), 293-299.

Hespanha, J., \& Morse, A. (1999). Stability of switched systems with average dwell-time. In Proceedings of the 38th IEEE Conference on Decision and Control (CDC) (pp. 2655-2660), New York, USA.

Ishii, H., \& Francis, B. (2001). Stabilizing a linear system by switching control with dwell time. In Proceedings of the American Control Conference (ACC) (pp. 1876-1881), Arlington, USA.
Koru, A. T., Delibaşı, A., \& Özbay, H. (2018). Dwell time-based stabilisation of switched delay systems using free-weighting matrices. International Journal of Control, 91(1), 1-11.

Li, Z., Gao, H., Agarwal, R., \& Kaynak, O. (2013). $H_{\infty}$ control of switched delayed systems with average dwell time. International Journal of Control, 86(12), 2146-2158.

Lin, H., \& Antsaklis, P. J. (2009). Stability and stabilizability of switched linear systems: A survey of recent results. IEEE Transactions on Automatic Control, 54(2), 308-322.

Liu, K., \& Fridman, E. (2012). Wirtinger's inequality and Lyapunov-based sampled-data stabilization. Automatica, 48(1), 102-108.

Liu, K., Seuret, A., \& Xia, Y. (2017). Stability analysis of systems with time-varying delays via the second-order Bessel-Legendre inequality. Automatica, 76, 138-142.

Mahmoud, M. S. (2010). Switched time-delay systems. Boston: SpringerVerlag.

Mitra, S., \& Liberzon, D. (2004). Stability of hybrid automata with average dwell time: An invariant approach. In Proceedings of the 43rd IEEE Conference on Decision and Control (CDC)(pp. 1394-1399), Nassau, Bahamas.

Park, P., Ko, J. W., \& Jeong, C. (2011). Reciprocally convex approach to stability of systems with time-varying delays. Automatica, 47(1), 235-238.

Prajna, S., Papachristodoulou, A., \& Parrilo, P. A. (2002). Introducing SOSTOOLS: A general purpose sum of squares programming solver. In Proceedings of the 41st IEEE Conference on Decision and Control (CDC) (pp. 741-746), Las Vegas, USA.

Seuret, A., \& Gouaisbaut, F. (2013). Wirtinger-based integral inequality: Application to time-delay systems. Automatica, 49(9), 2860-2866.

Seuret, A., \& Gouaisbaut, F. (2014). Complete quadratic Lyapunov functionals using Bessel-Legendre inequality. In European Control Conference (ECC)(pp. 448-453), Strasbourg, France.

Seuret, A., Gouaisbaut, F., \& Fridman, E. (2013). Stability of systems with fast-varying delay using improved Wirtinger's inequality. In Proceedings of the 52nd IEEE Conference on Decision and Control (CDC) (pp. 946-951), Florence, Italy.

Sturm, J. F. (1999). Using SeDuMi 1.02, a Matlab toolbox for optimization over symmetric cones. Optimization Methods and Software, 11(1-4), 625-653.

Sun, Z., \& Ge, S. (2011). Stability theory of switched dynamical systems. London, UK: Springer-Verlag.

Sun, X.-M., Zhao, J., \& Hill, D. J. (2006). Stability and $L_{2}$-gain analysis for switched delay systems: A delay-dependent method. Automatica, 42(10), 1769-1774.

Wu, M., He, Y., \& She, J.-H. (2010). Stability analysis and robust control of time-delay systems. Berlin: Springer.

Xiang, W. (2015). On equivalence of two stability criteria for continuoustime switched systems with dwell time constraint. Automatica, 54(0), $36-40$

Yan, P., \& Özbay, H. (2008). Stability analysis of switched time delay systems. SIAM Journal on Control and Optimization, 47(2), 936-949.

Yan, P., Özbay, H., \& Şansal, M. (2014). Robust stabilization of parameter varying time delay systems by switched controllers. Applied and Computational Mathematics, 13, 31-45.

Yang, F., Zhang, H., Hui, G., \& Wang, S. (2012). Mode-independent fuzzy fault-tolerant variable sampling stabilization of nonlinear networked systems with both time-varying and random delays. Fuzzy Sets and Systems, 207, 45-63.

Yuan, C., \& Wu, F. (2015). Hybrid control for switched linear systems with average dwell time. IEEE Transactions on Automatic Control, 60(1), 240-245.

Zhao, X., Shi, P., Yin, Y., \& Nguang, S. K. (2017). New results on stability of slowly switched systems: A multiple discontinuous Lyapunov function approach. IEEE Transactions on Automatic Control, 62(7), 3502-3509.

Zhao, X., Yin, Y., Niu, B., \& Zheng, X. (2016). Stabilization for a class of switched nonlinear systems with novel average dwell time switching by T-S fuzzy modeling. IEEE Transactions on Cybernetics, 46(8), 1952-1957. 